\title{
Investigation on International Students' Ultimate Attainment in Chinese Structural Auxiliary Word "De" (的) Acquisition
}

\author{
YANG Hai-feng, ZHAN Yi-han \\ Wuhan University of Technology, Wuhan, China
}

\begin{abstract}
This article studies the situations of international students' ultimate attainment in Chinese structural auxiliary word “De" (的) acquisition through questionnaires. And we found whether international students can use all types attributive "De" (的) correctly is mainly affected by the following two factors: (1) Influenced by frequency of attribute type appears and (2) affected by its own clarity of grammatical rules. We also noticed that the international students who are able to discriminate the "De" (的) and "Di" (地) in the predicates position better than the "De" (的) and "Di" (地) in the objects position.
\end{abstract}

Keywords: structural auxiliary word "De" (的), second language acquisition, comparative analysis, analysis of the errors

\section{Introduction}

As the most common word in modern Chinese, "De" (的) is the best-studied one among function words (SHI, 2008). The further research in modern grammatical meaning, is derived from Discussion on De (的) by ZHU De-xi (1961), who devised a distinctive research method of dividing "De" into three types: (1) attached components in adverbial grammatical units; (2) attached components in adjective grammatical units; and (3) attached components in noun grammatical units. It is an extremely debatable opinion with support and opposition by many scholars (as cited in SHI, 2008).

We believe that "De" (的) is an attributive mark with functions of syntax, pragmatics, and rhythm. The differences of structural auxiliary words between Chinese and other languages can be shown not only in grammar, but also in semantics and pragmatics. Even though the functions of structural auxiliary words have been distinguished in linguistic domain, in which “De" (的), “Di” (地), and “De" (得) are considered as the marks of attributes, adverbial modifiers, and complements respectively (LIU, 2008). In practice, the three structural auxiliary words are not distinguished by people, especially "De" (的) and "Di" (地) (B. ZHANG \& Y.-S. ZHANG, 2002). Accordingly, it is common to see the three structural auxiliary words muddled in issues and novels, which makes it more demanding for international students to acquire them.

Compared with correct forms in semantics or syntax to refer to structural and semantic mistakes, the errors when international students utilised "De" (的), have been analysed and researched just from the perspective of teaching instead of learners' acquisition and discrimination, in the domain of Teaching Chinese as a Foreign

\footnotetext{
* Acknowledgements: Sponsored by Special Fund of Basic Scientific Research of Central Colleges (No. 2014-Ib-064) (中央高校 基本科研业务费专项资金资助，项目批准号: 2014-Ib-064).

YANG Hai-feng, a female, associate professor, Ph.D., Chinese Department, Wuhan University of Technology.

ZHAN Yi-han, a male, junior, undergraduate, Chinese Department, Wuhan University of Technology.
} 
Language (TCFL) (TONG, 1986; YANG, 1996). Therefore, it is necessary to specifically investigate the Chinese language learners' acquisition of structural auxiliary word "De" (的) based on the predecessors' achievements regarding "De" (的) in modern Chinese (LV, 1982; Chinese Department of Peking University, 1996; LIU, 2001; ZHANG, 1994; CUI, 2001).

Currently, observation upon the errors from second language learners is the common practice to investigate acquisition of a certain grammatical rule and the error corpus is mainly extracted from their daily conversation, essays, as well as homework. Such method focuses on unconscious acquisition. Sometimes, to specifically survey second language learners' acquisition of a certain grammatical rule, researchers tend to put them into the conscious environment, which means that researchers examine their acquisition of the certain grammatical rule by questionnaires on account of universal avoidance strategy during learning, that they replace a certain grammatical rule with a relatively more secure one. Attributive to the complexity of concealing and revealing in Chinese structural auxiliary word "De" (的), this paper has drafted second language learners' ultimate attainment through questionnaires in order to prevent the avoidance strategy and specifically survey the acquisition. The subjects are the primary international students in preparatory class in Wuhan University of Technology (WUT) ${ }^{1}$. Two aspects are mainly involved in the questionnaires: (1) international students' ultimate attainment of "De" (的) in a variety of attributes and (2) their capacity of discrimination among “De" (的), “Di” (地), and “De" (得). Thirty-three questionnaires have been got totally.

\section{International Students' Acquisition of "De" (的) in Variety of Attributes}

Questionnaires design. We designed the questionnaire with 50 tests, including fillings, true or false, multiple choices, and sequencings, to examine international students' grasp of Chinese structural auxiliary words, based on the principles of comprehensiveness, key points, and diverse types, after referencing some exercises in Lectures on Function Words in Modern Chinese, Analysis of 900 Wrong Chinese Sentences and Chinese Course (Revised Edition), coupled with study on Chinese language, error corpus in daily conversation, and HSK (Hanyu Shuiping Kaoshi) corpus. These questionnaires are divided into pre-intermediate copy and advanced copy according to students' level.

Fillings, the first section, were designed to examine the international students' ability to distinguish between “De" (的) and “Di” (地) as well as grasp of “De” (的), “Di” (地), and “De” (得), each five of them considering “De" (的), “Di” (地), and “De” (得) sequentially.

True or false, the second section with 10 problems extracted from the most common mistakes in their essays, required subjects to correct the mistakes, aiming at inspecting their application of structural auxiliary words, which was beneficial for us to draw relatively objective conclusions by reducing the avoidance to some extent.

The third section was multiple choices, with 15 little problems. Five problems related to "De" (的), while the others were involved in “Di" (地) and “De" (得). Among the latter, nouns or noun phrases, personal pronouns, monosyllabic adjectives, disyllabic adjectives, duplication of adjectives, verbs or verb phrases, and locality words were selected as the attributes.

Sequencings, the fourth section, mainly surveyed subjects' grasp of "De" (的) “Di" (地) and their positions in sentences in the case of multiple attributes, adverbials, and their combination.

\footnotetext{
${ }^{1}$ International students studying Chinese for a year in China.
} 


\section{Statistical and Analytical Materials of the Questionnaires}

Table 1 shows that the average error rate of "De" (的) in all different attributes from overseas students was $48.5 \%$. Furthermore, disyllabic adjectives, verbs or verb phrases, and locality words as the attributes were not grasped well, whose error rates were over $50 \%$ respectively, overtaking the average error rates, while monosyllabic adjectives, duplication of adjectives, and nouns or noun phrases as attributes were mastered better, whose error rates were less than $40 \%$, below the average error rates. We believe that it is mainly influenced by the two following factors.

Table 1

Error Rates About Whether De (的) Could Be Used in All Types of Attributes From Overseas Students in China

\begin{tabular}{|c|c|c|c|c|c|c|c|c|}
\hline & $\begin{array}{l}\text { Average } \\
\text { error rate }\end{array}$ & $\begin{array}{l}\text { Monosyllabic } \\
\text { adjectives as } \\
\text { attributes }\end{array}$ & $\begin{array}{l}\text { Disyllabic } \\
\text { adjectives as } \\
\text { attributes }\end{array}$ & $\begin{array}{l}\text { Duplication of } \\
\text { adjectives as } \\
\text { attributes }\end{array}$ & $\begin{array}{l}\text { Nouns or noun } \\
\text { phrases as } \\
\text { attributes }\end{array}$ & $\begin{array}{l}\text { Personal } \\
\text { pronouns as } \\
\text { attributes }\end{array}$ & $\begin{array}{l}\text { Verbs or verb } \\
\text { phrases as } \\
\text { attributes }\end{array}$ & $\begin{array}{l}\text { Locality } \\
\text { words as } \\
\text { attributes }\end{array}$ \\
\hline $\begin{array}{l}\text { The error } \\
\text { rates of De } \\
\text { (的)\% }\end{array}$ & 48.5 & 35.4 & 56.1 & 39.7 & 30.2 & 27.6 & 57.7 & 64.3 \\
\hline
\end{tabular}

Firstly, it is influenced by the occurrence rates of attributive types. Personal pronoun is always used as the attribute so that international students know it best for "De" (的) behind it. The error rate of it was just $27.6 \%$, much lower than other attributive types. On the contrary, the low occurrence rates of disyllabic adjectives, verb or verb phrases, and locality words as attributes, result in higher error rates, as international students are relatively unfamiliar to these attributes and their knowledge about them is not well commanded.

Secondly, it is affected by the clarity of grammatical rules. Adults' second language acquisition on grammar is more based on the acquisition of grammatical rules than children. Thereby, the clarity of grammatical rules affects adults' grasp of these rules directly. For nouns as attributes, the frequency of monosyllabic nouns as attributes is lower than that of disyllabic nouns as attributes. In theory, the command of the former should be poorer. The reason why the reality is completely opposite is that the grammatical rule about whether "De" (的) could be used after a single syllable noun as an attribute is specific. Nonetheless, when a disyllabic noun is used as an attribute, the grammatical rule is vague and sometimes it depends on whether the context-syllable is consistent. To avoid too many characters of "De" (的), there is an ellipsis of "De" (的) if it does not cause an ambiguity. For instance, there are not characters of "De" (的) in "Hong hu shui, da hai feng" of “Hong hu shui, lang da lang (洪湖水, 浪打浪)" nor “Ying mian chui guo lai da hai feng (迎面吹 过来大海风)” (HUANG \& LIAO, 2011). Evidently, the lack of explicit rules of disyllabic nouns as attributes causes international students low error rates of rare attributes and high error rates of common attributes.

\section{Discrimination Among “De” (的), “Di” (地), and “De” (得) of International Students}

The pronunciations of “De" (的), “D” (地), and “De” (得) are basically the same, read as "De" softly, however, they must be divided into three different characters on paper. Most scholars used to claim not to distinguish “De" (的), “Di” (地), and “De" (得). For instance, WU Zong-yuan (1984) and JIANG Sheng-li (2008) declared to replace “Di" (地) and “De" (得) with “De" (的), while SUN Jian-ru (1995) and LIU Xiao-yun (2003) claimed to merge "De" (的) with “Di" (地) as they are close to each other, both of which could be used in prejudiced constructions. And in some Chinese newspaper and novels, the three structural auxiliary words were not distinguished in some articles. Nevertheless, the majority of people nowadays, tend to distinguish them for clear division of work after many years of practice. 


\section{International Students' Discrimination Between "De” (的) and “Di” (地)}

Questionnaires design. For the usage terms, in general, "De" (的) is utilised in front of a noun or a noun phrase while "Di" (地) is utilised in front of a verb or a verb phrase, so overseas students can judge which character should be used in front of the centre word according to its part of speech. This approach, based on the part of speech of the centre word to judge whether use "De" (的) or "Di" (地), is not demanding for second language learners. Relatively speaking, overseas students are likely to be confused with whether "De" (的) or “Di" (地) should be used in the formula of an adjective or an adjective phrase + De (的)/Di (地) + a verb or a verb phrase. Decided by the component of the above formula in a sentence, "Di" (地) should be used, when it is in a position of predicate, while "De" (的) should be used when it is a position of object, which means that "Di" (地) in an adverbial-verb phrase demands to be converted into "De" (的) once there is a delexicalised verb or a preposition in front of the phrase. For example:

(1) Wo men re lie di (地) tao lun le zhe ge ji hua-wo men dui zhe ge ji hua jin xing le re lie de (的) tao lun.

(2) Wo men che di (地) jie jue le zhe ge wen ti-zhe ge wen ti zhong yu de dao le che di de (的) jie jue.

(3) Wo men yan li di (地) da ji le zhe xie piao fan zi-wo men bi xu gei piao fan zi yi yan li de (的) da ji.

The structural auxiliary word should be used properly in terms of the change of structural relationship in our output. Accordingly, for our focus, we designed the questionnaire, in which the formula of an adjective or an adjective phrase + De (的)/(地) + a verb or a verb phrase is in positions of predicate and object respectively to examine international students" discrimination between "De" (的) and "Di" (地).

\section{Statistical and Analytical Materials of the Questionnaires}

Table 2 provides the information that the international students' average error rate given the structure of an adjective or an adjective phrase $+\mathrm{De}$ (的)/Di (地) + a verb or a verb phrase was $27.1 \%$, and the error rate was $32.2 \%$ when "De" was used in objects positions, while the error proportion was $22.0 \%$ when it was in predict position, which indicates that the overseas students' ability to distinguish "De" (的) and "Di" (地) is much worse on the object position than predict position. The cause leading to the phenomenon probably depends on a verb or a verb phrase as a centre word. Since in Chinese teaching classes, teachers generally tell students that “De" (的) is utilised in front of a noun while "Di" (地) is used in front of a verb, and students usually obey the rule to distinguish "De" (的) and "Di" (地). Therefore, when the formula is in the position of object only with “De" (的), international students' capacity of discrimination between "De" (的) and "Di" (地) is fairly much worse. It also proves that adult students' acquisition of grammar is based on a certain rule and its generality and serviceability would affect students' command of the grammatical point. Hence, Chinese teachers should tell students some slight differences properly instead of broad solutions when they teach students the difference between "D" (的) and "Di" (地). Only in this way can students master the knowledge quicker and better.

Table 2

International Students' Ability to Distinguish “De” (的) and “Di” (地)

\begin{tabular}{|c|c|c|c|}
\hline & Average error rate & Positions of objects & Positions of predicates \\
\hline $\begin{array}{l}\text { Error rates caused by muddling between } \\
\text { "De" (的) with "Di" (地) }\end{array}$ & $27.1 \%$ & $32.2 \%$ & $22.0 \%$ \\
\hline
\end{tabular}

International Students' Discrimination Between “De” (的) and “De” (得)

For the functional distinction of “De" (的) and “De" (得), the former is the mark of attributes, while the 
latter is the symbol of complements. And for the rules, "De" (的) is used for modification and restriction in front of nominals, while “De" (得) is used for supplements in back of predicates, e.g.,

(4) Wo ba gang xie wan de (的) yi pian wen zhang di gei le ba ba.

(5) Fei ji fei de (得) hen di.

Accordingly, we designed the questionnaire with the formula of a verb/adjective $+\mathrm{De}+\mathrm{a}$ noun or a verb/adjective + De + a complement respectively to inspect the overseas students' discrimination between "De" (的) and “De" (得).

\section{Statistical and Analytical Materials of the Questionnaires}

The statistics in Table 3 demonstrate that the international students' average error percentage of discrimination between “De" (的) and “De" (得) is 16.7\%, 10\% lower than that of discrimination between "De" (的) and "Di" (地) showed in Table 2, which reflects that their discrimination between them is better than that of “De" (的) and “Di” (地), probably because “De" (的) differs from “De" (得) more clearly. Moreover, Table 3 illustrates that their ability to distinguish "De" (的) and “De" (得) in the positions of attribute and complement does not differ markedly, whose error rates are approximately equal, because of predicates (either verbs or adjectives), which is the common component included in the attribute or the complement. It could be formalised a predict + De (的) + a noun or a predict + De (得) + a complement to judge to use “De" (的) or “De" (得). Second language learners can purely judge and determine to use “De" (的) or “De" (得) according to positions of structural auxiliary words relative to predicates.

Table 3

International Students' Ability to Distinguish “De” (的) and “De” (得)

\begin{tabular}{|c|c|c|c|}
\hline & Average error rate & Positions of attributes & Positions of complements \\
\hline $\begin{array}{l}\text { Error rates caused by muddling between } \\
\text { "De" (的) with “De" (得) }\end{array}$ & $16.8 \%$ & $24.3 \%$ & $20.0 \%$ \\
\hline
\end{tabular}

\section{Conclusion}

In conclusion, we may both illuminate the reasons leading to errors as well as enhance memory and help them notice the features of target language in semantic regions, affiliated semanteme, sememe systems, and syntagmatic relations, according to features of adult learners and grammatical rules, when “De” (的), “Di” (地), and “De" (得), the confused easily structural auxiliary words are distinguished in TCFL.

\section{References}

Chinese Department of Peking University (1995 and 1997 grades). (1996). Illustration of function words in modern Chinese (pp. 151-157). Shanghai: Commercial Press.

CUI, X. L. (2001). Concealing and revealing of De (的) when personal pronouns modify nouns (pp. 90-101). Beijing: Beijing Language and Culture University Press.

HUANG, B. R., \& LIAO, X. D. (2011). Modern Chinese (pp. 66-67). Beijing: Higher Education Press.

JIANG, S. L. (2008). Whether 的、地、得 can be regarded as one structural particle. Journal of Huangshan University, 10(6), 101-104.

LIU, D. Q. (2008). Typological features of nominal phrases in Chinese. Studies of the Chinese Language, 1, 3-20.

LIU, X. Y. (2003). The exchange usage of structural auxiliary words "de" \& “di". Journal of Shanxi Radio \& TV University, 2 , $87-88$.

LIU, Y. H. (2001). Modern Chinese grammar. Shanghai: Commercial Press.

LV, S. X. (1982). 800 words in modern Chinese (pp. 132-143). Shanghai: Commercial Press. 
SUN, J. R. (1995). Splitting and integration of auxiliary words De (的). Journal of Yangzhou Teachers College (Social Science Edition), 4, 80-82.

SHI, D. X. (2008). De (的) and De (的) construction. Contempory Linguistic, 4, 298-307.

TONG, H. J. (1986). Analysis of wrong sentenses when foreigners learn Chinese (pp. 164-211). Beijing: Beijing Language and Culture University Press.

WU, Z. Y. (1984). De (的), Di (地) and De (得), The structural auxiliary words should be merged. Journal of Ningxia University (Social Science Edition), 3, 72-75.

YANG, Q. H. (1996). Difficulties analysis in TCFL (pp. 226-255). Beijing: Beijing Normal University Press.

ZHANG, B., \& ZHANG, Y. S. (2002). Modern Chinese function words (pp. 195-200). Shanghai: East China Normal University Press.

ZHANG, G. X. (1994). Syntactic, semantic and pragmatic analysis of De (的). Journal of Huaibei Teachers College, 3, $100-107$.

ZHU, D. X. (1961). Discussion on De (的). Studies of the Chinese Language, 12, 1-15. 